\title{
Multi-keV Analyses of a Presolar Mg-Silicate Grain via SEM/STEM
}

\author{
Laura Seifert ${ }^{1}$, Pierre Haenecour $^{1}$, Tom Zega ${ }^{1,2}$, Christine Floss ${ }^{3}$, Jane Howe $^{4}$ \\ 1. Lunar and Planetary Laboratory, University of Arizona, Tucson, USA \\ 2. Materials Science and Engineering, University of Arizona, Tucson, USA \\ 3. Laboratory for Space Sciences and Physics Department, Washington University, St. Louis, USA \\ 4. Hitachi High-Technologies America Inc., Clarksburg, USA
}

Presolar grains are particles of dust that condensed in circumstellar envelopes and the ejecta of stellar explosions [1]. These grains, preserved in primitive meteorites, were transported through the interstellar medium and became the building blocks of our solar system. Studying these materials can provide information about stellar nucleosynthesis, circumstellar thermodynamics, and processing in our own solar system [1-2]. Here we discuss the structure and chemistry of a Mg-silicate presolar grain from the type 3.0 CO Chondrite Dominion Range (DOM) 08006. Presolar silicates are important to study because they are among the most abundant types of dust in the interstellar medium [3].

Carbonaceous and O-rich grains were identified via raster-ion-imaging using NanoSIMS at Washington University in St. Louis in a petrographic thin section of DOM 08006 [4]. We chose one grain, DOM-59 for detailed analysis using transmission electron microscopy (TEM). An electron transparent section of DOM-59 was prepared with an FEI Helios G3 focused-ion-beam-scanning-electron microscope (FIBSEM) at the Lunar and Planetary Laboratory (LPL) using previously described techniques [5]. The FIB section was analyzed using the aberration-corrected Hitachi HF5000 TEM at 200kV and the newly installed Hitachi SU9000 SEM/STEM at 10 and $30 \mathrm{kV}$. Both instruments are equipped with secondary electron (SE) detectors, scanning TEM (STEM)-based bright-field (BF) and dark-field (DF) imaging detectors, as well as Oxford Instruments energy-dispersive X-ray spectrometer (EDS) system.

NanoSIMS mapping shows a crescent shaped O-isotopic anomaly with enrichment in ${ }^{17} \mathrm{O}$ and depletion in ${ }^{18} \mathrm{O}$, placing it into the field of Group 2 grains [2]. The shape of DOM-59, as revealed from SE imaging in the SEM, is roughly round and measures $310 \times 390 \mathrm{~nm}$. TEM-EDS $(512 \mathrm{px} \times 512 \mathrm{px})$ maps were obtained at $200 \mathrm{kV}$, revealing a Mg-silicate grain containing a core-shell structure. Selected-area electron- diffraction (SAED) patterns from across the grain indicate that DOM-59 is crystalline and consistent with a pyroxene. The core of the grain is dominated by Ca-rich pyroxene, while the surrounding shell is Ca-poor and $\mathrm{Mg}$-rich pyroxene. EDS mapping at $200 \mathrm{kV}$ also reveals an iron rich region surrounding the $\mathrm{Mg}$-silicate shell.

Additional measurements were made at lower voltages $(10 \mathrm{kV}, 30 \mathrm{kV})$ to study the calcium core and iron-rich regions outside the grain. BF and DF images of DOM-59 at 30kV reveal a distinct line of contrast at the top of the grain, indicated by red arrows in Fig. 1. The contrast seems to be anticorrelated with $\mathrm{Mg}$, while the Ca-rich core occurs on the line, as seen at both $10 \mathrm{kV}$ and $30 \mathrm{kV}$ (Fig. 2). The SE image at $30 \mathrm{kV}$ confirms the $\mathrm{Ca}$ core is at the center of the grain and is not a surface feature. Analysis of the iron rich regions revealed iron oxides are distributed through the silicate matrix surrounding the grain. Low $\mathrm{kV}$ imaging shows a distinct rim around the grain that is consistent with a $\mathrm{Mg}$-silicate.

Group 2 grains, like DOM-59, were long thought to originate in low-mass $(<2 \mathrm{M} \odot)$ asymptotic giant 
branch (AGB) stars that experienced deep-mixing processes [2]. However, new work suggests that these grains may have originated in intermediate mass (4 to $8 \mathrm{M}_{\odot}$ ) AGB stars that did not experience deep mixing [6]. Equilibrium thermodynamic calculations constrain solids which condense out of solar composition gas at different temperatures and pressures [7]. Comparing DOM-59 to these calculations, the Ca-rich pyroxene core condensed first at 1347K, followed by the $\mathrm{Mg}$-rich pyroxene shell at $1316 \mathrm{~K}$, assuming a total pressure of $10^{-4}$ bars.

We hypothesize that the core-shell structure in DOM-59 was produced by the Ca-rich core condensing first and acting as a nucleation site for the surrounding Mg-rich shell. The Fe visible in the EDS maps (Fig. 2) is not well constrained. Additional measurements of surrounding matrix can confirm if Fe is only found in regions surrounding the grain or if it is seen elsewhere in the matrix of the meteorite. This grain was extracted from a pristine meteorite, and so it should not have experienced parent-body processing, therefore making it unlikely that $\mathrm{Fe}$ diffused through the matrix as a result of such processing $[8,9]$.

\section{References}

[1] E Zinner in "Treatise on Geochemistry”, Ed. H Holland, K Turekian (Elsevier Ltd, Waltham, p.181.

[2] L Nittler et al., The Astrophysical Journal, 682 (2008), p.1450.

[3] B Draine, Annu. Rev. Astron. Astrophys., 41, (2003), p. 241.

[4] P Haenecour et al., Geochimica et Cosmochimica Acta 221, (2018), p.379.

[5] T Zega et al., Meteoritics and Planetary Science 42, (2007), p.1373.

[6] M Lugaro et al. Nature Astronomy 1, (2017), p.27.

[7] K Lodders, The Astrophysical Journal 591, (2003), p.1220.

[8] L Nittler et al., Geochimica et Cosmochimica Acta (2018) in press.

[9] We recognize NASA grants \#NNX12AL47G, \#NNX15AJ22G and NSF grants \#1531243, \#0619599 for funding instrumentation in the Kuiper Materials Imaging and Characterization Facility at LPL.

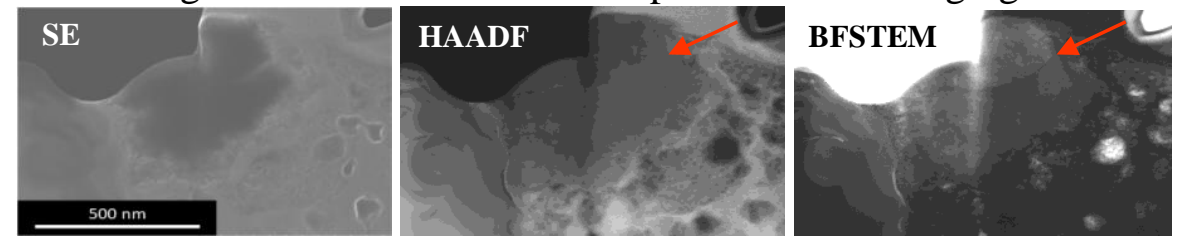

Figure 1. SE, HAADF, BFSTEM images of DOM-59 at $30 \mathrm{kV}$.

$10 \mathrm{kV}$

$30 \mathrm{kV}$

$200 \mathrm{kV}$

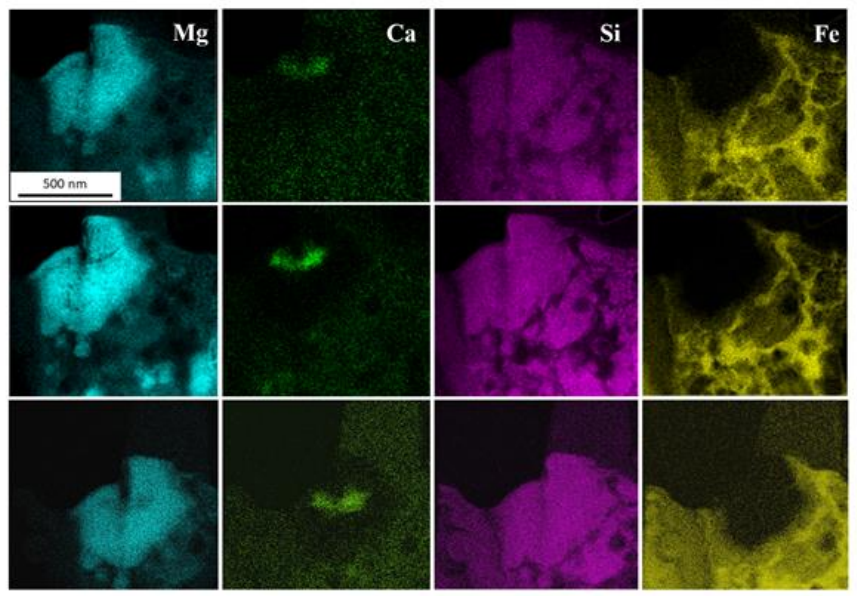

Figure 2. EDS maps of Mg, Ca, Si, Fe from DOM-59 at $10 \mathrm{kV}, 30 \mathrm{kV}$ and $200 \mathrm{kV}$. 